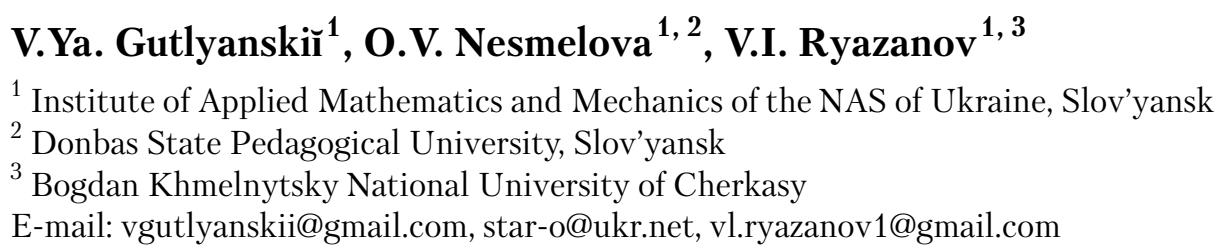

\title{
On the quasilinear Poisson equations in the complex plane
}

Presented by Corresponding Member of the NAS of Ukraine V.Ya. Gutlyanskiu

First, we study the existence and regularity of solutions for the linear Poisson equations $\Delta U(z)=g(z)$ in bounded domains $D$ of the complex plane $\mathbb{C}$ with charges $g$ in the classes $L^{1}(D) \cap L_{\mathrm{loc}}^{p}(D), p>1$. Then, applying the Leray-Schauder approach, we prove the existence of Hölder-continuous solutions $U$ in the class $W_{\text {loc }}^{2, p}(D)$ for the quasilinear Poisson equations of the form $\Delta U(z)=h(z) \cdot f(U(z))$ with $h$ in the same classes as $g$ and continuous functions $f: \mathbb{R} \rightarrow \mathbb{R}$ such that $f(t) / t \rightarrow 0$ as $t \rightarrow \infty$. These results can be applied to various problems of mathematical physics.

Keywords: potential theory, quasilinear Poisson equations, semilinear equations, anisotropic and inhomogeneous media, quasiconformal mappings.

1. Introduction. The study of elliptic partial differential equations in two dimensions by the methods of complex analysis and quasiconformal mappings with applications to nonlinear elasticity, gas flow, hydrodynamics, and other sections of natural science has been initiated by M.A. Lavrentiev, L. Bers, L. Nirenberg, I.N. Vekua, B. Bojarski, and others (see, e.g., [1-5] and the references therein). A rather comprehensive treatment of the present state of the theory is given in the excellent book of K. Astala, T. Iwaniec, and G. Martin [6].

In series of our recent papers (see, e.g., $[7,8]$ ), we have proposed another application of the theory of quasiconformal mappings to the the study of semilinear partial differential equations of the form

$$
\operatorname{div}[A(z) \nabla u(z)]=f(u), \quad z \in D, \quad D \subseteq \mathbb{C},
$$

the diffusion term of which is the divergence-form elliptic operator $L(u)$, whereas its reaction term $f(u)$ is a non-linear function. Here, the symmetric matrix function $A(z)=\left\{a_{i j}(z)\right\}$,

(C) V.Ya. Gutlyanskiĭ, O.V. Nesmelova, V.I. Ryazanov, 2020 
$\operatorname{det} A(z)=1$, with measurable real-valued entries satisfies the uniform ellipticity condition

$$
\frac{1}{K}|\xi|^{2} \leqslant\langle A(z) \xi, \xi\rangle \leqslant K|\xi|^{2} \quad \text { a.e. in } \quad D, \quad 1 \leqslant K<\infty
$$

for every $\xi \in \mathbb{R}^{2}$. The set of all such matrix functions is denoted by $M_{K}^{2 \times 2}(D)$.

It was shown that, by the chain rule for the composition function $u=U \circ \omega$, where $\omega$ is a quasiconformal mapping that agrees with the matrix function $A(z)$, the following basic formula holds:

$$
\operatorname{div}[A(z) \nabla(U(\omega(z)))]=J_{\omega}(z) \Delta U(\omega(z)),
$$

where $J_{\omega}(z)$ stands for the Jacobian of the mapping $\omega$.

Namely, this formula (understood in the sense of distributions) takes place for all $U \in W_{\text {loc }}^{1,2}(G)$, $A \in M_{K}^{2 \times 2}(D)$ and quasiconformal homeomorphisms $\omega: D \rightarrow G$ satisfying the Beltrami equation

$$
\omega_{\bar{z}}(z)=\mu(z) \omega_{z}(z) \text { a.e. in } D,
$$

where its complex dilatation

$$
\mu(z)=\frac{a_{22}-a_{11}-2 i a_{12}}{\operatorname{det}(I+A)}
$$

satisfies the uniform ellipticity condition

$$
|\mu(z)| \leqslant \frac{1+K}{1-K}
$$

Thus, the study of semilinear equations of the form (1) in domains $D$ of a finite area can be reduced in a suitable way to the corresponding investigations of the quasilinear Poisson equations of the form $\Delta U=h \cdot f(U)$. The latter is the subject of the present paper.

Below, $\mathbb{D}$ denotes the unit disk $\{z \in \mathbb{C}:|z|<1\}$ in the complex plane $\mathbb{C}, \mathbb{D}_{R}\left(z_{0}\right):=\left\{z \in \mathbb{C}:\left|z-z_{0}\right|<R\right\}$ for $z_{0} \in \mathbb{C}$ and $R \in(0, \infty), \mathbb{D}_{R}:=\mathbb{D}_{R}(0)$.

2. Basic facts from the potential theory. For the sake of completeness, we repeat the fundamental results concerning the potential theory in a plane, given in [8], and strengthen some of them.

First of all, we recall that, by Corollary 1 in [8], if, for every Borel set $B$ in $\mathbb{C}$,

$$
v(B):=\int_{B} g(z) d m(z)
$$

where $g: \mathbb{C} \rightarrow \mathbb{R}$ is an integrable function with compact support, then

$$
\Delta N_{g}=g
$$

where

$$
N_{g}(z):=\frac{1}{2 \pi} \int_{\mathbb{C}} \ln |z-w| g(w) d m(w),
$$


in the distributional sense, i.e.,

$$
\int_{\mathbb{C}} N_{g}(z) \Delta \psi(z) d m(z)=\int_{\mathbb{C}} \psi(z) g(z) d m(z) \quad \psi \in C_{0}^{\infty}(\mathbb{C}) \theta .
$$

Here, the function $g$ is called a density of the charge $v$, and the function $N_{g}$ is said to be the Newtonian potential of $g$.

Recall also the definition of the formal complex derivatives:

$$
\frac{\partial}{\partial z}:=\frac{1}{2}\left\{\frac{\partial}{\partial x}-i \frac{\partial}{\partial y}\right\}, \quad \frac{\partial}{\partial z}:=\frac{1}{2}\left\{\frac{\partial}{\partial x}+i \frac{\partial}{\partial y}\right\}, \quad z=x+i y .
$$

The elementary algebraic calculations show their relation to the Laplacian

$$
\Delta:=\frac{\partial^{2}}{\partial x^{2}}+\frac{\partial^{2}}{\partial y^{2}}=4 \frac{\partial^{2}}{\partial z \partial \bar{z}}=4 \frac{\partial^{2}}{\partial \bar{z} \partial z}
$$

Further, we apply the theory of the well-known integral operators

$$
\operatorname{Tg}(z):=\frac{1}{\pi} \int_{\mathbb{C}} g(w) \frac{d m(w)}{z-w}, \quad \bar{T} g(z):=\frac{1}{\pi} \int_{\mathbb{C}} g(w) \frac{d m(\omega)}{\bar{z}-\bar{\omega}}
$$

defined for integrable functions with a compact support $K$ and studied in detail.

The following theorem on the Newtonian potential strengthens Theorem 2 from [8]. It is important to obtain solutions of a higher regularity to the Poisson equations (8), as well as to the corresponding semilinear equations.

Here and later on, given a domain $D$ in $\mathbb{C}$, a function $g: D \rightarrow \mathbb{R}$ is assumed to be extended onto $\mathbb{C}$ by zero outside of $D$.

Theorem 1. Let $D$ be a bounded domain in $\mathbb{C}$. Suppose that $g \in L^{1}(D)$. Then $N_{g} \in W_{\text {loc }}^{1, q}(\mathbb{C})$ for all $q \in[1,2)$, and there exist the generalized derivatives by Sobolev $\frac{\partial^{2} N_{g}}{\partial z \partial \bar{z}}$ and $\frac{\partial^{2} N_{g}}{\partial \bar{z} \partial z}$ and

$$
4 \frac{\partial^{2} N_{g}}{\partial z \partial \bar{z}}=\Delta N_{g}=4 \frac{\partial^{2} N_{g}}{\partial \bar{z} \partial z}=g \text { a.e. in } \mathbb{C} .
$$

Moreover, $N_{g} \in L_{\mathrm{loc}}^{s}(\mathbb{C})$ for all $s \in[1, \infty)$. More precisely,

$$
\left\|N_{g}\right\|_{s} \leqslant\|g\|_{1} \cdot\|\ln \mid \xi\|_{s} \quad \forall s \in[1, \infty)
$$

where $\left\|N_{g}\right\|_{s}$ is in $\mathbb{D}_{r}$ for all $r \in(0, \infty)$, and $\|\ln \mid \xi\|_{s}$ is in $\mathbb{D}_{R+r}$ if $D \subseteq \mathbb{D}_{R}$.

If $g \in L^{p}(D)$ for some $p \in(1,2]$, then $N_{g} \in W_{\mathrm{loc}}^{2, p}(\mathbb{C})$ and

$$
N_{g} \in W_{\text {loc }}^{1, \gamma}(\mathbb{C}) \quad \forall \gamma \in(1, q) \text {, where } q=2 p /(2-p)>2 \text {. }
$$

In addition, the collection $\left\{N_{g}\right\}$ is locally $\beta$-Hölder equicontinuous in $\mathbb{C}$ for all $\beta \in(0,1-2 / q)$, and the collection $\left\{N_{g}^{\prime}\right\}$ of its first partial derivatives is strictly compact in $L^{\gamma}(D)$ for all $\gamma \in(1, q)$, if the collection $\{g\}$ is bounded in $L^{p}(D)$. 
Finally, if $g \in L^{p}(D)$ for some $p>2$, then $N_{g} \in C_{\mathrm{loc}}^{1, \alpha}(\mathbb{C})$ with $\alpha=(p-2) / p$. Furthermore, the collection $\left\{N_{g}^{\prime}\right\}$ is locally Hölder-equicontinuous in $\mathbb{C}$ with the given $\alpha$, if $\{g\}$ is bounded in $L^{p}(D)$.

Proof. Note that $N_{g}$ is the convolution $\psi * g$, where $\psi(\xi)=\ln |\xi|$. Hence, (14) follows (e.g., by Corollary 4.5.2 in [9]). Moreover, $\frac{\partial \psi * g}{\partial z}=\frac{\partial \psi}{\partial z} * g$ and $\frac{\partial \psi * g}{\partial \bar{z}}=\frac{\partial \psi}{\partial \bar{z}} * g$ (see, e.g., (4.2.5) in [9]). By elementary calculations,

$$
\frac{\partial}{\partial z} \ln |z-w|=\frac{1}{2} \frac{1}{z-w}, \quad \frac{\partial}{\partial \bar{z}} \ln |z-w|=\frac{1}{2} \frac{1}{\bar{z}-\bar{\omega}} .
$$

Consequently,

$$
\frac{\partial N_{g}(z)}{\partial z}=\frac{1}{4} \operatorname{Tg}(z), \quad \frac{\partial N_{g}(z)}{\partial \bar{z}}=\frac{1}{4} \bar{T} g(z) .
$$

Thus, the rest conclusions for $g \in L^{1}(D)$ follow by Theorems $1.13-1.14$ in [5].

Next, if $g \in L^{p}(D)$ with $p \in(1,2]$, then $N_{g} \in W_{\text {loc }}^{1, \gamma}(\mathbb{C})$ for all $\gamma \in(1, q)$, where $q=2 p /$ ' $(2-p)>2$, by Theorem 1.27, (1.27) in [5] and, moreover, $N_{g} \in W_{\text {loc }}^{2, p}(\mathbb{C})$ by Theorems 1.36-1.37 in [5]. In addition, a collection $\left\{N_{g}\right\}$ is locally $\beta$-Hölder-equicontinuous in $\mathbb{C}$ for all $\beta \in(0,1-2 / q)$ (see, e.g., Lemma 2.7 in [3]), and the collection $\left\{N_{g}^{\prime}\right\}$ of its first partial derivatives is strictly compact in $L^{\gamma}(D)$ for all $\gamma \in(1, q)$, if the collection $\{g\}$ is bounded by the norm in $L^{p}(D)$ (see, e.g., Theorem 1.4.3 in [10] and Theorem 1.27 in [5]).

The last item of Theorem 1 was derived in Theorem 2 from [8].

Remark 1. Note, generally speaking, that $N_{g} \notin W_{\text {loc }}^{2,1}$ and $N_{g} \notin C$, if $g \in L^{1}(\mathbb{C})$ (see, for instance, Example 7.5 in [11], and the example in Remark 2 from [8]).

Corollary 1. Let $D$ be a subdomain of $\mathbb{D}, g: D \rightarrow \mathbb{R}$ be in $L^{1}(D)$ and in $L_{\mathrm{loc}}^{p}(D)$ for some $p>1$. Then $N_{g} \in W_{\mathrm{loc}}^{2, p}(D)$ and satisfies (13) a.e. in D. Moreover, $N_{g} \in W_{\mathrm{loc}}^{1, q}(D)$ for $q>2$, and $N_{g}$ is locally Hölder-continuous in D. Furthermore, $N_{g} \in C_{\operatorname{loc}}^{1, \alpha}(D)$ with $\alpha=(p-2) / p$, if $g \in L_{\mathrm{loc}}^{p}(D)$ for $p>2$.

In addition, the collection $\left\{N_{g}\right\}$ is locally $\beta$-Hölder-equicontinuous in $D$ for all $\beta \in$ $\in(0,1-2 / q)$, and the collection $\left\{N_{g}^{\prime}\right\}$ of its first partial derivatives is strictly compact in $L_{\mathrm{loc}}^{\gamma}(D)$ for all $\gamma \in(1, q)$, if a collection $\{g\}$ is bounded in $L^{1}(D)$ and in $L_{\mathrm{loc}}^{p}(D)$ for some $p \in(1,2]$, where $q$ is defined in (15).

Finally, the collection $\left\{N_{g}^{\prime}\right\}$ is locally $\alpha$-Hölder-equicontinuous in $D$ with the given $\alpha$, if $a$ collection $\{g\}$ is bounded in $L^{\mathrm{P}}(D)$ and in $L_{\mathrm{loc}}^{p}(D)$ for $p>2$.

Proof. Given $z_{0} \in D$ and $0<R<\operatorname{dist}\left(z_{0}, \partial D\right), N_{g}=N_{g_{1}}+N_{g_{2}}$ with $g_{2}:=g-g_{1}$ and $g_{1}:=g \cdot \chi$, where $\chi$ is the characteristic function of the disk $\mathbb{D}_{R}\left(z_{0}\right)$. The first summand satisfies all desired properties by Theorem 1 , and the second one is a harmonic function in $\mathbb{D}_{R}\left(z_{0}\right)$ (see, e.g., Theorem 3.1.2 in [12]). Thus, the first part follows. Under the proof of the rest part, the same decomposition is applied. However, in the case we need the following 2 explicit estimates for the second summand in a smaller disk $\mathbb{D}_{r}\left(z_{0}\right), r \in(0, R)$,

$$
\left|N_{g_{2}}\left(z_{2}\right)-N_{g_{2}}\left(z_{1}\right)\right| \leqslant\left|\int_{z_{1}}^{z_{2}} \frac{\partial N_{g_{2}}}{\partial z} d z\right|+\left|\int_{z_{1}}^{z_{2}} \frac{\partial N_{g_{2}}}{\partial \bar{z}} d \bar{z}\right| \leqslant \frac{1}{2 \pi} \frac{\|g\|_{1}}{(R-r)}\left|z_{2}-z_{1}\right|
$$


and, since the function $T g_{2}$ is analytic in $\mathbb{D}_{r}\left(z_{0}\right)$ and the function $\bar{T} g_{2}=\overline{T g_{2}}$ (for the real-valued function $\left.g_{2}\right)$ is anti-analytic in $\mathbb{D}_{r}\left(z_{0}\right)$, similarly,

$$
\left|N_{g_{2}}^{\prime}\left(z_{2}\right)-N_{g_{2}}^{\prime}\left(z_{1}\right)\right| \leqslant \frac{1}{4}\left|\int_{z_{1}}^{z_{2}} \frac{\partial T g_{2}}{\partial z} d z\right| \leqslant \frac{1}{4 \pi} \frac{\|g\|_{1}}{(R-r)^{2}}\left|z_{2}-z_{1}\right| .
$$

Here, we denote, by $N_{g_{2}}^{\prime}$, any of the first partial derivatives of $N_{g_{2}}$ (see (11)):

$$
\frac{\partial}{\partial x}=\frac{\partial}{\partial z}+\frac{\partial}{\partial \bar{z}}, \frac{\partial}{\partial y}=i \cdot\left(\frac{\partial}{\partial z}-\frac{\partial}{\partial \bar{z}}\right), \quad z=x+i y,
$$

take relation (16) into account, and calculate the given integrals over the segment $\left[z_{1}, z_{2}\right] \subset D_{r}\left(z_{0}\right)$ of the straight line going through $z_{1}, z_{2} \in \mathbb{D}_{r}\left(z_{0}\right)$.

3. On the solvability of quasilinear Poisson equations. In this section, we study the solvability problem for quasilinear Poisson equations of the form $\Delta U=h f(U)$. The well-known Leray-Schauder approach allows us to reduce the problem to the study of the corresponding linear Poisson equation from the previous section.

Theorem 2. Let $h: \mathbb{C} \rightarrow \mathbb{R}$ be a function in the class $L^{p}(\mathbb{C})$ for $p>1$ with compact support. Suppose that a function $f: \mathbb{R} \rightarrow \mathbb{R}$ is continuous and

$$
\lim _{t \rightarrow \infty} \frac{f(t)}{t}=0 .
$$

Then there is a continuous function $U: \mathbb{C} \rightarrow \mathbb{R}$ in the class $W_{\text {loc }}^{2, p}(\mathbb{C})$ such that

$$
\Delta U(z)=h(z) f(U(z)) \quad \text { a.e. }
$$

and $U=N_{g}$, where $g: \mathbb{C} \rightarrow \mathbb{R}$ is a function in $L^{p}$ whose support is in the support of $h$, and the upper bound of $\|g\|_{p}$ depends only on $\|h\|_{p}$ and on the function $f$. Moreover, $U \in W_{\text {loc }}^{1, q}(\mathbb{C})$ for some $q>2$, and $U$ is locally Hölder-continuous. Furthermore, $U \in C_{\text {loc }}^{1, \alpha}(\mathbb{C})$ with $\alpha=(p-2) / p$, if $p>2$.

In particular, $U \in C_{\text {loc }}^{1, \alpha}(\mathbb{C})$ for all $\alpha=(0,1)$, if $h$ is bounded in Theorem 2 .

Proof. If $\|h\|_{p}=0$ or $\|f\|_{c}=0$, then any constant function $U$ in $\mathbb{C}$ gives the desired solution of (19). Thus, we may assume that $\|h\|_{p} \neq 0$ and $\|f\|_{c} \neq 0$. Set $f_{*}(s)=\max _{|t| \leqslant s}|f(t)|, s \in \mathbb{R}^{+}:=[0, \infty)$. Then the function $f_{*}: \mathbb{R}^{+} \rightarrow \mathbb{R}^{+}$is continuous and nondecreasing. Moreover, $f_{*}(s) / s \rightarrow 0$ as $s \rightarrow \infty$ by (18).

By Theorem 1 in [8], we obtain a family of operators $F(g ; \tau): L_{h}^{p}(\mathbb{C}) \rightarrow L_{h}^{p}(\mathbb{C}), L_{h}^{p}(\mathbb{C})$ is the subspace of $L^{p}(\mathbb{C})$ with supports in the support of $h$,

$$
F(g ; \tau):=\tau h \cdot f\left(N_{g}\right) \quad \forall \tau \in[0,1]
$$

which satisfies all groups of hypotheses H1-H3 of Theorem 1 in [13]. Indeed:

H1. First of all, $F(g ; \tau): L_{h}^{p}(\mathbb{C})$ for all $\tau \in[0,1]$ and $g \in L_{h}^{p}(\mathbb{C})$, because, by Theorem 1 in [8], the function $f\left(N_{g}\right)$ is continuous and

$$
\|F(g ; \tau)\|_{p} \leqslant\|h\|_{p} f_{*}\left(M\|g\|_{p}\right)<\infty \quad \forall \tau \in[0,1],
$$


where $M$ is the constant from estimate (19) in [8]. Thus, by Theorem 1 from [8] in combination with the Arzela-Ascoli theorem (see, e.g., Theorem IV.6.7 in [14]), the operators $F(g ; \tau)$ are completely continuous for every $\tau \in[0,1]$ and even uniformly continuous in the parameter $\tau \in[0,1]$.

H2. The index of the operator $F(g ; 0)$ is obviously equal to 1 .

H3. By Theorem 1 in [8], we have, for solutions of the equations $g=F(g ; \tau)$ :

$$
\|g\|_{p} \leqslant\|h\|_{p} f_{*}\left(M\|g\|_{p}\right),
$$

i.e.,

$$
\frac{f_{*}\left(M\|g\|_{p}\right)}{M\|g\|_{p}} \geqslant \frac{1}{M\|h\|_{p}} .
$$

Hence, $\|g\|_{p}$ is bounded in the parameter $\tau \in[0,1]$ by condition (18).

Thus, by Theorem 1 in [13], there is a function $g \in L_{h}^{p}(D)$ with $F(g ; 1)=g$, and, by Theorem 1, the function $U:=N_{g}$ gives the desired solution of (19).

Theorem 3. Let $D$ be adomain in $\mathbb{D}, h: D \rightarrow \mathbb{R}$ bein $L^{1}(D) \cap L_{\text {loc }}^{p}(D), p>1$, andlet $f: \mathbb{R} \rightarrow \mathbb{R}$ satisfy the hypothesis of Theorem 2. Then there is a weak solution $U: D \rightarrow \mathbb{R}$ of the quasilinear Poisson equation (19) in the class $C(D) \cap W_{\mathrm{loc}}^{1, q}(D)$ for some $q>2$ which is locally Hölder-continuous in D .

Proof. Let $D_{k}$ be an expanding sequence of domains in $\mathbb{C}$ with $\overline{D_{k}} \subset D, k=1,2, \ldots$, exhausting $D$, i.e. $\bigcup_{k=1}^{\infty} D_{k}=D$. Let us extend $h$ by zero outside of $D$. Set $h_{k}=h \chi_{k}$, where $\chi_{k}$ is a characteristic function of $D_{k}$ in $\mathbb{C}$, and $U_{k}=N_{g_{k}}$, where $g_{k}$ corresponds to $h_{k}$ by Theorem 2. Now, by Corollary 1, the family of functions $\left\{N_{g_{k}}\right\}$ is Hölder-equicontinuous on each $D_{m}, m=1,2, \ldots$.

Moreover, by Theorems 1 and 2, as well as by the Hölder integral inequality, we have

$$
\left\|N_{g_{k}}\right\|_{L^{1}\left(\mathbb{D}_{\varepsilon}\right)} \leqslant\left\|g_{k}\right\|_{1} \cdot\left\|\ln \left|\xi\left\|_{L^{1}\left(\mathbb{D}_{1+\varepsilon}\right)} \leqslant \pi^{1-\frac{1}{p}} \Psi\left(\|h\|_{p}\right) \cdot\right\| \ln \right| \xi\right\|_{L^{1}\left(\mathbb{D}_{1+\varepsilon}\right)}
$$

for small enough $\varepsilon>0$ such that $\mathbb{D}_{\varepsilon} \subset D_{1}$, where the function $\Psi: \mathbb{R}^{+} \rightarrow \mathbb{R}^{+}$depends only on $f$. Hence, for each $k=1,2, \ldots$, there is a point $z_{k} \in \mathbb{D}_{\varepsilon}$ with $\left|N_{g_{k}}\left(z_{k}\right)\right| \leqslant c:=\pi^{\frac{p-1}{p}} \Psi\left(\|h\|_{p}\right) \cdot\|\ln |\xi|\|_{L^{1}\left(\mathbb{D}_{1+\varepsilon}\right)} / \pi \varepsilon^{2}$, because, by (22), the mean integral value of $\left|N_{g_{k}}\left(z_{k}\right)\right|$ over the disk $\mathbb{D}_{\varepsilon}$ cannot be greater than the given number $c$. Combining the latter fact with Hölder-equicontinuity of the sequence $N_{g_{k}}, k=1,2, \ldots$, on each $D_{m}, m=1,2, \ldots$, we obtain also its boundedness. Thus, by the ArzelaAscoli theorem (see, e.g., Theorem IV.6.7 in [14]), the family of functions $\left\{N_{g_{k}}\right\}$ is compact on each $D_{m}, m=1,2, \ldots$.

Without loss of generality, we may assume that $p \in(1,2]$. Then, by Corollary 1 , the Newtonian potential $\left\{N_{g_{k}}\right\}, m=1,2, \ldots$, is in the class $W_{\text {loc }}^{1, q}$ for some $q>2$, and the family $\left\{N_{g_{k}}^{\prime}\right\}$ is also compact on each $D_{m}, m=1,2, \ldots$ by the norm of $L^{q}$. Consequently, the sequence $\left\{N_{g_{k}}\right\}$ is compact on each $D_{m}, m=1,2, \ldots$ by any norm $\|\cdot\|$ of $W^{1, q}$, too (see, e.g., Theorem 2.5.1 in [15]).

Next, let us apply the so-called diagonal process. Namely, let $U_{k}^{(1)}, k=1,2, \ldots$, be a subsequence of $\left\{N_{g_{k}}\right\}$ that converges uniformly to a function $U: D_{1} \rightarrow \mathbb{R}$ by the norm $\|\cdot\|$ on the 
domain $D_{1}$. Of course, we may assume that $\left\|U_{k}^{(1)}-U\right\|_{C}<1 / k$, as well as $\left\|U_{k}^{(1)}-U\right\|<1 / k$ for all $k=1,2, \ldots$. Similarly, a subsequence $U_{k}^{(2)}$ of $U_{k}^{(1)}$ with respect to the domain $D_{2}$ is defined. Let us continue the process by induction and, finally, consider the diagonal subsequence $U_{m}:=U_{m}^{(m)}, m=1,2, \ldots$ of the sequence $N_{g_{i}}$.

It is clear by the construction that $\left.U_{m}^{k}\right|_{D}$ converges to a function $U: D \rightarrow \mathbb{R}$ locally uniformly and also in $W_{\mathrm{loc}}^{1, q}(D), q>2$. Thus, $U \in C(D) \cap W_{\mathrm{loc}}^{1, q}(D)$, and, consequently, $U$ is locally Hölder-continuous in $D$. Moreover, $U$ is a weak solution of Eq. (19) in the domain $D$. Indeed, by (10) and the definition of generalized derivatives, we have that $U_{m}$ satisfy the relations

$$
\int_{D}\left\langle\nabla U_{m}(z), \nabla \psi(z)\right\rangle d m(z)+\int_{D} h_{m}(z) f\left(U_{m}(z)\right) \psi(z) d m(z)=0 \quad \forall \psi \in C_{0}^{\infty}(D) .
$$

Passing to the limit as $m \rightarrow \infty$, we obtain the desired conclusion.

This work was partially supported by grants of Ministry of Education and Science of Ukraine, project number is $0119 U 100421$.

\section{REFERENCES}

1. Bers, L. \& Nirenberg, L. (1954, August). On a representation theorem for linear elliptic systems with discontinuous coefficients and its applications. Convegno Internazionale sulle equazioni lineari alle derivate parziali, Trieste (pp. 111-140). Rome: Edizioni Cremonese.

2. Bojarski, B. V. (1955). Homeomorphic solutions of Beltrami systems. Dokl. Akad. Nauk SSSR (N.S.), 102, pp. 661-664 (in Russian).

3. Bojarski, B. \& Iwaniec, T. (1983). Analytical foundations of the theory of quasiconformal mappings in $\mathbb{R}^{n}$. Ann. Acad. Sci. Fenn. Ser. A. I. Math., 8, No. 2, pp. 257-324.

4. Lavrentiev, M. (1938). Sur une critère différentiel des transformations homéomorphes des domains à trois dimensions. Dokl. Acad. Nauk. SSSR, 20, pp. 241-242.

5. Vekua, I. N. (1962). Generalized analytic functions. Oxford, New York: Pergamon Press.

6. Astala, K., Iwaniec, T. \& Martin, G. (2009). Elliptic partial differential equations and quasiconformal mappings in the plane. Princeton Mathematical Series (Vol. 48). Princeton, NJ: Princeton University Press.

7. Gutlyanskii, V. \& Nesmelova, O. \& Ryazanov, V. (2018). On quasiconformal maps and semilinear equations in the plane. J. Math. Sci., 229, No. 1, pp. 7-29.

8. Gutlyanskii, V. Ya., Nesmelova, O. V. \& Ryazanov, V. I. (2018). On the regularity of solutions of quasilinear Poisson equations. Dopov. Nac. akad. nauk Ukr., No. 10, pp. 9-17. https://doi.org/10.15407/dopovidi2018.10.009

9. Hörmander, L. (1983). The analysis of linear partial differential operators. I. Distribution theory and Fourier analysis. Grundlehren der Mathematischen Wissenschaften. (Vol. 256). Berlin: Springer.

10. Sobolev, S. L. (1991). Some applications of functional analysis in mathematical physics. Mathematical Monographs. (Vol. 90). Providence, RI: AMS.

11. Giaquinta, M. \& Martinazzi, L. (2012). An introduction to the regularity theory for elliptic systems, harmonic maps and minimal graphs. 2nd ed. Lecture Notes (Scuola Normale Superiore) (Vol. 11). Pisa: Edizioni della Normale.

12. Ransford, T. (1995). Potential theory in the complex plane. London Mathematical Society Student Texts. (Vol. 28). Cambridge: Cambridge University Press.

13. Leray, J. \& Schauder, Ju. (1934). Topologie et équations fonctionnelles. Ann. Sci. Ecole Norm. Sup., 51, No. 3, pp. 45-78.

14. Dunford, N. \& Schwartz, J.T. (1958). Linear operators. Pt. I. General theory. Pure and Applied Mathematics. (Vol. 7). New York: Interscience.

15. Mihlin, S. G. (1977). Linear partial differential equations. Moscow: Vysshaya Shkola (in Russian).

Received 20.10.2019 
В.Я. Гутлянський ${ }^{1}$, О.В. Несмелова ${ }^{1,2}$, В.І. Рязанов ${ }^{1,3}$

${ }^{1}$ Інститут прикладної математики і механіки НАН України, Слов'янськ

2 Донбаський державний педагогічний університет, Слов'янськ

${ }^{3}$ Черкаський національний університет ім. Богдана Хмельницького

E-mail:vgutlyanskii@gmail.com, star-o@ukr.net,vl.ryazanov1@gmail.com

\section{ПРО КВАЗІЛІНІЙНІ РІВНЯННЯ ПУАССОНА В КОМПЛЕКСНІЙ ПЛОЩИНІ}

Вивчено існування і регулярність розв'язків для лінійних рівнянь Пуассона виду $\Delta U(z)=g(z)$ в обмежених областях $D$ комплексної площини $\mathbb{C}$ із зарядами $g$ в класах $L^{1}(D) \cap L_{\mathrm{loc}}^{p}(D), p>1.3$ використанням підходу Лере-Шаудера доведено існування неперервних за Гельдером розв’язків $U$ в класі $W_{\text {loc }}^{2, p}(D)$ для квазілінійних рівнянь Пуассона виду $\Delta U(z)=h(z) \cdot f(U(z))$ з $h$ із того самого класу, що і $g$, та неперервними функціями $f: \mathbb{R} \rightarrow \mathbb{R}$ такими, що $f(t) / t \rightarrow 0$ при $t \rightarrow \infty$. Отримані результати можуть бути застосовані до різномантіних задач математичної фізики.

Ключові слова: теорія потенціалу, квазілінійні рівняння Пуассона, напівлінійні рівняння, анізотропні та неоднорідні середовища, квазіконформні відображення.

\section{В.Я. Гутлянский}

О.В. Несмелова ${ }^{1,2}$, В.И. Рязанов ${ }^{1,3}$

${ }^{1}$ Институт прикладной математики и механики НАН Украины, Славянск

2 Донбасский государственный педагогический университет, Славянск

3 Черкасский национальный университет им. Богдана Хмельницкого

E-mail: vgutlyanskii@gmail.com, star-o@ukr.net,vl.ryazanov1@gmail.com

\section{О КВАЗИЛИНЕЙНЫХ УРАВНЕНИЯХ ПУАССОНА В КОМПЛЕКСНОЙ ПЛОСКОСТИ}

Изучено существование и регулярность решений для линейних уравнений Пуассона вида $\Delta U(z)=g(z)$ в ограниченных областях $D$ комплексной плоскости $\mathbb{C}$ с зарядами $g$ в классах $L^{1}(D) \cap L_{\mathrm{loc}}^{p}(D), p>1$. C применением подхода Лере-Шаудера доказано существование непрерывных по Гёльдеру решений $U$ в классе $W_{\mathrm{loc}}^{2, p}(D)$ для квазилинейных уравнений Пуассона вида $\Delta U(z)=h(z) \cdot f(U(z))$ с $h$ из того же класса, что и $g$, и непрерывными функциями $f: \mathbb{R} \rightarrow \mathbb{R}$ такими, что $f(t) / t \rightarrow 0$ при $t \rightarrow \infty$. Полученные результаты могут быть применены к различным задачам математической физики.

Ключевые слова: теория потенциала, квазилинейные уравнения Пуассона, полулинейные уравнения, анизотропные и неоднородные среды, квазиконформные отображения. 